\title{
Evaluation of inhomogeneities of repolarization in patients with psoriasis vulgaris
}

Korhan Soylu' ${ }^{1}$, Sinan Inci², Gökhan Aksan³, Gökay Nar ${ }^{2}$, Esra Pancar Yüksel', Hande Serra Ocal', Mustafa Çapraz ${ }^{6}$, Serkan Yüksel ${ }^{1}$, Mahmut Şahin ${ }^{1}$

\author{
${ }^{1}$ Department of Cardiology, Faculty of Medicine, Ondokuz Mayis University, Samsun, \\ Turkey \\ 2Department of Cardiology, Aksaray State Hospital, Aksaray, Turkey \\ ${ }^{3}$ Department of Cardiology, Gazi State Hospital, Samsun, Turkey \\ ${ }^{4}$ Department of Dermatology, Faculty of Medicine, Ondokuz Mayis University, \\ Samsun, Turkey \\ ${ }^{5}$ Department of Dermatology, Gazi State Hospital, Samsun, Turkey \\ ${ }^{6}$ Department of Internal Medicine, Faculty of Medicine, Amasya University, Amasya, \\ Turkey
}

Submitted: 15 October 2014

Accepted: 8 January 2015

Arch Med Sci 2016; 12, 6: 1225-1231

DOI: 10.5114 /aoms.2016.62922

Copyright $\odot 2016$ Termedia \& Banach

\section{Abstract}

Introduction: The arrhythmia potential has not been investigated adequately in psoriatic patients. In this study, we assessed the ventricular repolarization dispersion, using the Tp-e interval and the Tp-e/QT ratio, and investigated the association with inflammation.

Material and methods: Seventy-one psoriasis vulgaris patients and 70 ageand gender-matched healthy individuals were enrolled in the study. The severity of the disease was calculated using Psoriasis Area and Severity Index scoring. The QTd was defined as the difference between the maximum and minimum QT intervals. The Tp-e interval was defined as the interval from the peak of the T wave to the end of the T wave. The Tp-e interval was corrected for heart rate. The Tp-e/QT ratio was calculated using these measurements. Results: There were no significant differences between the groups with respect to basal clinical and laboratory characteristics $(p>0.05)$. The Tp-e interval, the corrected Tp-e interval (cTp-e) and the Tp-e/QT ratio were also significantly higher in psoriasis patients compared to the control group $(78.5 \pm 8.0 \mathrm{~ms}$ vs. $71.4 \pm 7.6 \mathrm{~ms}, p<0.001,86.3 \pm 13.2 \mathrm{~ms}$ vs. $77.6 \pm 9.0 \mathrm{~ms}$, $p<0.001$ and $0.21 \pm 0.02$ vs. $0.19 \pm 0.02, p<0.001$ respectively). A significant correlation was detected between the cTp-e time and the Tp-e/QT ratio and the PASI score in the group of psoriatic patients $(r=0.51, p<0.001 ; r=0.59$, $p<0.001$, respectively).

Conclusions: In our study, we detected a significant increase in the Tp-e interval and the Tp-e/QT ratio in patients with psoriasis vulgaris. The Tp-e interval and the Tp-e/QT ratio may be predictors for ventricular arrhythmias in patients with psoriasis vulgaris.

Key words: psoriasis vulgaris, Tp-e interval, Tp-e/QT ratio.

\section{Introduction}

Psoriasis is a chronic and inflammatory skin disease that is observed in approximately $1-3 \%$ of the overall population $[1,2]$. While it is basically characterized by its dermatological nature, the effects of the sys-

\author{
Corresponding author: \\ Korhan Soylu MD, \\ Department of Cardiology \\ Faculty of Medicine \\ Ondokuz Mayis University \\ 55139, Samsun, Turkey \\ Phone: +90 3623121919 \\ (ext: 3249) \\ Fax: +903624577146 \\ E-mail: korhansoylu@yahoo. \\ com
}


temic inflammation may involve the whole body and the cardiovascular system. Studies have revealed increased rates of cardiovascular disorders including hypertension, atherosclerosis and cardiac valve diseases in patients with psoriasis $[3,4]$. On the other hand, the effects of chronic inflammation on the cardiac conduction system and the arrhythmia potential have not been adequately investigated. A limited number of studies have reported an increased QT dispersion and its potential as a predictor for a probable arrhythmia in psoriasis patients $[5,6]$. However, the effect of prolonged ventricular repolarization indices on arrhythmogenic risk and sudden cardiac death (SCD) in these patients has not yet been clarified.

QT dispersion (QTd), corrected QT dispersion (cQTd) and Tp-e (transmural dispersion of repolarization) are well-established indicators of myocardial repolarization abnormalities [7, 8]. The time from the $T$ wave peak to the end of the $T$ wave (Tp-e) on ECG, and the ratio of this time to the QT interval (Tp-e/QT) are newly identified parameters for myocardial repolarization dispersion [8, 9]. An increased Tp-e interval was associated with increased cardiovascular mortality and ventricular tachyarrhythmia [10-12]. In addition, the Tp-e/QT ratio was claimed to be more valuable than the QTd, cQTd, Tp-e parameters in assessing the ventricular repolarization dispersion [13, 14]. In this study, the ventricular repolarization dispersion was evaluated using the Tp-e interval and the Tp-e/QT ratio in patients with psoriasis vulgaris, and its association with inflammation was investigated.

\section{Material and methods}

\section{Study population}

Seventy-one psoriasis vulgaris patients, who presented to Ondokuz Mayıs University and Gazi State Hospital Dermatology Clinic between May 2013 and January 2014 (diagnosed via punch biopsy), and 70 age- and gender-matched healthy individuals were included in the study. Patients with hypertension $(\mathrm{HT})$, diabetes mellitus (DM), history of coronary artery disease (CAD), left ventricular (LV) wall motion abnormality, an LV ejection fraction (LVEF) less than 50\%, primary cardiomyopathy, valvular heart disease, bundle branch block and atrioventricular conduction abnormalities on ECG, non-sinus rhythm, thyroid dysfunction, left ventricular hypertrophy on transthoracic echocardiography, anemia, renal failure, pulmonary disease, electrolyte imbalance, ECGs without clearly analysable QT segment, and patients taking class I-IV antiarrhythmic drugs, digitalis, tricyclic antidepressants, antipsychotics, or antihistaminics were excluded from the study.

\section{Biochemical measurements}

Biochemical parameters were measured using commercial kits and the Abbott ARCHITECT c8000 (Abbott Laboratories, USA) auto-analyzer. Hematologic parameters were measured with the Abbott CellDyn 3700 (Abbott Laboratories, USA) device via the laser and impedance method. The immunonephelometric method (BN ProSpec System protein analyzer, Siemens Healthcare Diagnostics, Deerfield, IL, USA) was used for measurement of high-sensitivity C-reactive protein (hs-CRP).

\section{Disease severity}

The severity of the disease was evaluated by the Psoriasis Area and Severity Index (PASI) (range: 0-72). Extent of the skin lesions located on the head, trunk, and upper and lower limbs was assessed (score range for each location: 0-6). The following features of the skin lesions were evaluated: erythema (0-4 points), infiltration (0-4 points), and desquamation (0-4 points) [15].

\section{Electrocardiography}

12-lead ECG was performed at a rate of $50 \mathrm{~mm} / \mathrm{s}$ and $20 \mathrm{~mm} / \mathrm{mV}$ amplitude following a 10-minute rest with the patient in the supine position (Nihon Kohden, Tokyo, Japan). ECG measurements of QT and Tp-e intervals were performed by two cardiologists, who were blinded to the patient data. To decrease the error measurements, QT and Tp-e intervals were measured manually with calipers and a magnifying glass. An average value of three readings was calculated for each lead. The QT interval was measured based on the interval between the start of the QRS complex and the end of the T wave on lead DII, and was corrected for the heart rate using Bazett's formula (i.e., QTc = $\mathrm{QT} / \sqrt{\mathrm{R}}-\mathrm{R}$ interval). The QTd was defined as the difference between the maximum and minimum QT intervals of the 12 leads [16]. The Tp-e interval was defined as the interval from the peak of the $T$ wave to the end of the $T$ wave. Measurements of Tp-e interval were performed from the precordial leads [10]. The Tp-e interval was corrected for heart rate. The Tp-e/QT ratio was calculated using these measurements. Inter-observer and intra-observer coefficients of variation were $3.1 \%$ and $2.8 \%$, respectively.

\section{Standard echocardiography}

Echocardiography investigations were performed by an experienced cardiologist, who was unaware of the patient's clinical status, in the left lateral decubitus position, using a GE Vingmed Vivid 7 (GE Vingmed Ultrasound, Horten, Norway) echocardiography device. Obtaining the paraster- 
nal long axis, short axis, apical four-chamber and two-chamber images, the assessment was performed using the M-mode, 2D, continuous wave Doppler, pulse wave Doppler method based on the American Society of Echocardiography criteria [17]. The values were measured on three separate beats, and then averaged for all parameters. Left ventricular mass and its index (LVMI) were measured as described by Devereux et al. using the equation previously presented [18]. Accordingly, LVMI was compared to body surface area. Left ventricular mass and its index values higher than $125 \mathrm{~g} / \mathrm{m}^{2}$ in men and $110 \mathrm{~g} / \mathrm{m}^{2}$ in women were regarded as indicating the presence of left ventricular hypertrophy (LVH).

\section{Statistical analysis}

All parameters were expressed as mean \pm standard deviation. All data were analyzed using SPSS for Windows version 15.0 software (Chicago, IL,
USA). Categorical variables were presented as frequencies and percentages, while the continuous variables were expressed as means and SD. The normal distribution of continuous variables was tested with the Kolmogorov-Smirnov test. For continuous variables, the independent samples $t$-test or Mann Whitney $U$-test was used as appropriate. Any correlation between the data was tested with the Spearman and Pearson correlation analysis. Comparison of the categorical values was carried out by the $\chi^{2}$ test. A value of $p<0.05$ was considered to be significant.

\section{Results}

The study enrolled 141 patients in total, including 71 patients with psoriasis vulgaris and 70 healthy individuals. The basal clinical and laboratory characteristics of the patients are presented in Table I. There was no significant difference between the groups in age, gender,

Table I. Baseline clinical and laboratory characteristics of study population and comparison between groups

\begin{tabular}{|c|c|c|c|}
\hline Parameter & Psoriasis group $(n=71)$ & Control group $(n=70)$ & $P$-value \\
\hline Age [years] & $44.8 \pm 13.2$ & $43.4 \pm 12.4$ & 0.533 \\
\hline Male, $n(\%)$ & $40(56)$ & $35(50)$ & 0.541 \\
\hline Body mass index $\left[\mathrm{kg} / \mathrm{m}^{2}\right]$ & $26.9 \pm 3.9$ & $27.7 \pm 3.5$ & 0.191 \\
\hline Systolic blood pressure [mm Hg] & $126.5 \pm 8.8$ & $126.8 \pm 7.7$ & 0.881 \\
\hline Diastolic blood pressure [mm Hg] & $73.8 \pm 6.9$ & $72.3 \pm 6.4$ & 0.198 \\
\hline Heart rate [beats/min] & $72 \pm 6.9$ & $71.1 \pm 5.7$ & 0.406 \\
\hline Hemoglobin [g/dl] & $13.8 \pm 1.6$ & $13.3 \pm 1.7$ & 0.098 \\
\hline White blood cell count $\left[\times 10^{3 /} \mathrm{mm}^{3}\right]$ & $7.09 \pm 1.7$ & $7.2 \pm 1.7$ & 0.822 \\
\hline Neutrophils $\left[\times 10^{3 /} \mathrm{mm}^{3}\right]$ & $4.36 \pm 1.64$ & $4.24 \pm 1.51$ & 0.670 \\
\hline Platelets $\left[\times 10^{3 /} \mathrm{mm}^{3}\right]$ & $236.4 \pm 57.4$ & $227.5 \pm 50.9$ & 0.339 \\
\hline Creatinine $[\mathrm{mg} / \mathrm{dl}]$ & $0.84 \pm 0.16$ & $0.81 \pm 0.16$ & 0.347 \\
\hline Fasting glucose [mg/dl] & $87.5 \pm 10.3$ & $86.7 \pm 10.1$ & 0.617 \\
\hline AST [U/I] & $23 \pm 11.4$ & $22.4 \pm 10.5$ & 0.729 \\
\hline $\operatorname{ALT}[\mathrm{U} / \mathrm{l}]$ & $22.1 \pm 11.3$ & $20.1 \pm 10.9$ & 0.283 \\
\hline Total cholesterol [mg/dl] & $191.7 \pm 38.3$ & $188.6 \pm 40$ & 0.638 \\
\hline Low-density lipoprotein cholesterol [mg/dl] & $118.5 \pm 30.4$ & $122.1 \pm 27.4$ & 0.473 \\
\hline High-density lipoprotein cholesterol [mg/dl] & $49.2 \pm 9.4$ & $50.2 \pm 9.3$ & 0.535 \\
\hline Triglyceride [mg/dl] & $151.4 \pm 59.4$ & $145.5 \pm 68.2$ & 0.591 \\
\hline $\mathrm{Na}[\mathrm{mmol} / \mathrm{l}]$ & $140.9 \pm 2.6$ & $141 \pm 2.8$ & 0.853 \\
\hline $\mathrm{K}[\mathrm{mmol} / \mathrm{l}]$ & $4.2 \pm 0.3$ & $4.2 \pm 0.3$ & 0.548 \\
\hline $\mathrm{Ca}[\mathrm{mg} / \mathrm{dl}]$ & $9 \pm 0.6$ & $8.9 \pm 0.6$ & 0.375 \\
\hline $\mathrm{TSH}[\mu \mathrm{IU} / \mathrm{ml}]$ & $2.5 \pm 1.1$ & $2.4 \pm 1$ & 0.741 \\
\hline PASI & $5.75 \pm 2.1$ & & \\
\hline hs-CRP $[\mathrm{mg} / \mathrm{ll}]$ & $1.8 \pm 0.8$ & $1.0 \pm 0.8$ & $<0.001$ \\
\hline
\end{tabular}


body mass index (BMI), systolic and diastolic blood pressure, heart rate, lipid parameters, creatinine, glucose, white sphere count, hemoglobin, platelet count, serum electrolytes ( $\mathrm{Na}, \mathrm{K}$, $\mathrm{Ca})$ or the thyroid-stimulating hormone (TSH) values. However, hs-CRP levels were significantly higher in patients with psoriasis compared to controls (1.8 \pm 0.8 vs. $1.0 \pm 0.8 \mathrm{mg} / \mathrm{l}, p<0.001)$. While the mean PASI score was detected to be $5.75 \pm 2.1$ in the psoriatic patients, the mean disease duration was $129.8 \pm 71.4$ months (Table I).
The echocardiographic investigations revealed no significant differences between the groups $(p>$ 0.05 ) (Table II). There was no significant difference between the groups in LVM or LVMI $(145.4 \pm 39.8$ vs. $141.2 \pm 43.4 \mathrm{~g}$ and $76 \pm 20.2$ vs. $\left.74 \pm 18.9 \mathrm{~g} / \mathrm{m}^{2}\right)$.

Electrocardiographic parameters of the groups are shown in Table III. The maximum corrected QT (cQTmax), QTd, and cQTd times were significantly higher in the psoriasis patient group compared to the control group $(409.2 \pm 22.6$ vs. $399.8 \pm 20.8 \mathrm{~ms}$, $p=0.012,31.4 \pm 6.1$ vs. $25.9 \pm 5.9 \mathrm{~ms}, p<0.001$

Table II. Conventional echocardiographic parameters

\begin{tabular}{|c|c|c|c|}
\hline Parameter & Psoriasis group $(n=71)$ & Control group $(n=70)$ & $P$-value \\
\hline LVEDD $[\mathrm{mm}]$ & $47.0 \pm 3.4$ & $46.4 \pm 3.2$ & 0.323 \\
\hline LVESD $[\mathrm{mm}]$ & $26.5 \pm 3.4$ & $25.9 \pm 3.9$ & 0.281 \\
\hline IVS thickness [mm] & $9.4 \pm 1.4$ & $9.3 \pm 1.3$ & 0.625 \\
\hline PW thickness [mm] & $9.0 \pm 1.1$ & $8.9 \pm 1.1$ & 0.767 \\
\hline LA dimension [mm] & $34.3 \pm 3.4$ & $33.8 \pm 4.2$ & 0.484 \\
\hline LVEF (\%) & $58.9 \pm 4.8$ & $60.2 \pm 4.2$ & 0.111 \\
\hline RV dimension [mm] & $22.4 \pm 3.4$ & $21.9 \pm 3.3$ & 0.365 \\
\hline $\mathrm{sPAP}[\mathrm{mm} \mathrm{Hg}]$ & $24.9 \pm 5.0$ & $24.8 \pm 5.0$ & 0.981 \\
\hline DT [ms] & $181.3 \pm 24.1$ & $175.9 \pm 30$ & 0.244 \\
\hline IVRT [ms] & $82.3 \pm 12.6$ & $79.7 \pm 13.1$ & 0.228 \\
\hline$E[\mathrm{~m} / \mathrm{s}]$ & $0.77 \pm 0.20$ & $0.78 \pm 0.18$ & 0.602 \\
\hline$A[\mathrm{~m} / \mathrm{s}]$ & $0.72 \pm 0.18$ & $0.77 \pm 0.17$ & 0.073 \\
\hline$E / A$ & $1.2 \pm 0.3$ & $1.1 \pm 0.2$ & 0.102 \\
\hline LVM [g] & $145.4 \pm 39.8$ & $141.2 \pm 43.4$ & 0.265 \\
\hline LVMI $\left[\mathrm{g} / \mathrm{m}^{2}\right]$ & $76 \pm 20.2$ & $74 \pm 18.9$ & 0.232 \\
\hline
\end{tabular}

IVS - ventricular septal thickness, PW - posterior wall thickness, LVEDD - left ventricular end-diastolic dimension, LVESD - left ventricular end-systolic dimensions, LA - left atrium, LVEF - left ventricular ejection fraction, RV - right ventricular, SPAP - systolic pulmonary artery pressure, DT - deceleration time, IVRT - isovolumetric relaxation time, E - early diastolic flow, A - atrial contraction signal, LVM - left ventricular mass, LVMI - left ventricular mass index.

Table III. Electrocardiographic findings of the groups

\begin{tabular}{|c|c|c|c|}
\hline Parameter & Psoriasis group $(n=71)$ & Control group $(n=70)$ & $P$-value \\
\hline QTmax [ms] & $369.4 \pm 15.0$ & $368.9 \pm 14.1$ & 0.833 \\
\hline cQTmax [ms] & $409.2 \pm 22.6$ & $399.8 \pm 20.8$ & 0.012 \\
\hline QTmin [ms] & $339.4 \pm 14.5$ & $342.9 \pm 13.1$ & 0.139 \\
\hline cQTmin [ms] & $376.7 \pm 19.9$ & $372.5 \pm 20.2$ & 0.222 \\
\hline QTd [ms] & $31.4 \pm 6.1$ & $25.9 \pm 5.9$ & $<0.001$ \\
\hline cQTd [ms] & $34.1 \pm 7.7$ & $27.3 \pm 5.7$ & $<0.001$ \\
\hline Tp-e [ms] & $78.5 \pm 8.0$ & $71.4 \pm 7.6$ & $<0.001$ \\
\hline cTp-e [ms] & $86.3 \pm 13.2$ & $77.6 \pm 9.0$ & $<0.001$ \\
\hline Tp-e/QT & $0.21 \pm 0.02$ & $0.19 \pm 0.02$ & $<0.001$ \\
\hline
\end{tabular}

QTmax - QT maximum, cQTmax - corrected QT maximum, QTmin - QT minimum, cQTmin - corrected QT minimum, QTd - QT dispersion, cQTd - corrected QT dispersion, Tp-e - transmural dispersion of repolarization, cTp-e-corrected transmural dispersion of repolarization. 
and $34.1 \pm 7.7$ vs. $27.3 \pm 5.7 \mathrm{~ms}, p<0.001$ respectively). The Tp-e interval, the corrected Tp-e interval (cTp-e) and the Tp-e/QT ratio were also significantly higher in psoriasis patients compared to the control group (78.5 \pm 8.0 vs. $71.4 \pm 7.6 \mathrm{~ms}$, $p<0.001,86.3 \pm 13.2$ vs. $77.6 \pm 9.0 \mathrm{~ms}, p<0.001$ and $0.21 \pm 0.02$ vs. $0.19 \pm 0.02, p<0.001$, respectively).

There was a significant correlation between the cTp-e time and the Tp-e/QT ratio, and the PASI score in the group of psoriatic patients $(r=0.51$, $p<0.001$; and $r=0.59, p<0.001$, respectively) (Figures 1 and 2). Additionally, the cTp-e interval and the Tp-e/QT ratio were significantly correlated with the hs-CRP levels $(r=0.54, p<0.001$; and $r=$ $0.48, p<0.001$, respectively).

\section{Discussion}

In our study, we found that the patients with psoriasis vulgaris had a significantly prolonged Tp-e interval and Tp-e/QT ratio, compared to the controls. In addition, these electrocardiographic parameters were significantly correlated with the plasma hs-CRP level, reflecting the severity of the disease, and the PASI score.

Psoriasis vulgaris is a common, autoimmune, inflammatory skin disease [19]. Recent studies show an increased risk of cardiovascular disease in patients with psoriasis vulgaris [20-22]. While the association between development of arrhythmia and inflammation has been investigated, data on rhythm and conduction abnormalities are very limited in psoriatic patients [23]. In several studies, the heart rate, premature atrial and ventricular beat were reported to be increased in psoriatic

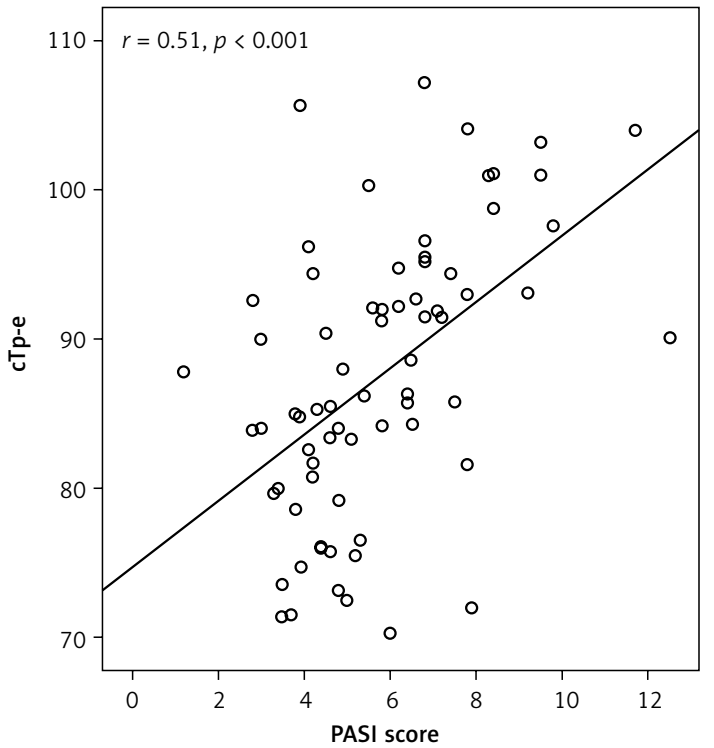

Figure 1. Scatter analysis of the correlation between the cTp-e time and the PASI score, in psoriatic patients patients [24, 25]. Moreover, there are data supporting the disturbances in heart rate variability and recovery times which develop on the basis of autonomic dysfunction in psoriasis $[26,27]$.

While the QT dispersion is considered as an indicator of the regional ventricular repolarization, increased values are associated with arrhythmia and sudden cardiac death $[28,29]$. Two previous studies demonstrated prolonged QTd and cQTd intervals in patients with psoriasis vulgaris [5, 6]. In our study, we also detected higher QTd and cQTd intervals compared to those in the control group. Still, the Tp-e and the Tp-e/QT parameters were not evaluated previously in psoriatic patients. Recent studies show that the Tp-e interval is an index of the total spatial dispersion of cardiac repolarization [8, 14]. Increased dispersion of repolarization is one of the several factors that may promote malignant ventricular arrhythmias [30]. Accordingly, a long Tp-e has been associated with malignant arrhythmias in the long QT syndrome [31], the Brugada syndrome [10], in hypertrophic cardiomyopathy [32] and in patients with myocardial infarction [12, 33]. However, it is affected by the variations of the heart rate and body weight. Tp-e/QT ratio was reported to be a more accurate marker for ventricular arrhythmogenesis compared to Tp-e interval and QTd, due to its independence from the heart rate [14, 33].

It has been demonstrated that Tp-e duration and prolongation of Tp-e/QT ratio are predictors of ventricular arrhythmia. In the study of Letsas et al. [34], they found higher Tp-e duration and $\mathrm{Tp}$-e/QT ratio in patients with Brugada syndrome, in whom ventricular tachycardia (VT)/ventricu-

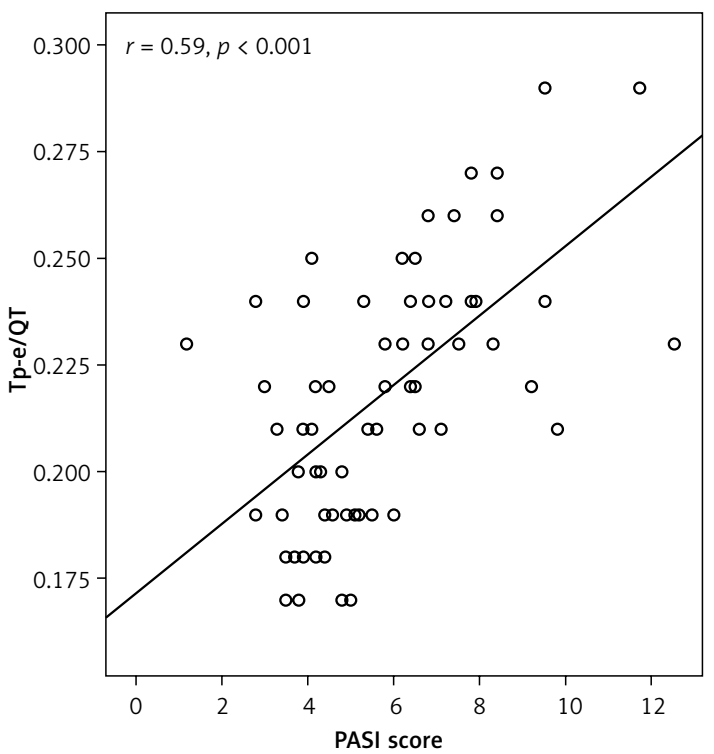

Figure 2. Scatter analysis of the correlation between the Tp-e/QT index and the PASI score in psoriatic patients 
lar fibrillation (VF) was induced in electrophysiological studies (EPS); when compared with the patients in which ventricular tachycardia (VT)/ ventricular fibrillation (VF) was not induced, the values were $88.82 \pm 15.76 \mathrm{~ms}$ and $0.227 \pm 0.034$ $\mathrm{ms}$, respectively. Similarly, in a long-term follow-up study performed by Castro Hevia et al. [10], they found that Tp-e duration $\geq 100$ ms in Brugada syndrome could predict recurrent life-threatening ventricular arrhythmias with $77.8 \%$ sensitivity and $70 \%$ specificity. In a long-term follow-up study by Watanabe et al. [13], in which patients with organic heart disease were investigated, Tp-e duration in patients in whom spontaneous VT had developed was $125 \pm 23.2$ ms and cTp-e duration was $132 \pm 37.4$ ms. Again, in the study of Zhao et al. [33], in which the relation between ventricular repolarization indices and prognosis of acute coronary syndrome (ACS) was investigated and in which the mean duration of follow-up was 17 months, they found that Tp-e/QT $\geq 0.29$ predicted cardiac death (cardiac failure, fatal arrhythmia and sudden death, and fatal myocardial infarction) with $83 \%$ sensitivity and $71 \%$ specificity. In the current study, lower ventricular repolarization indices were found in patients with psoriasis when compared with the abovementioned studies. The absence of organic heart disease in the psoriasis group might contribute to lower Tp-e and cTp-e duration and Tp-e/QT ratio.

The present study is the first to report the Tp-e interval and the Tp-e/QT rate. Studies performed to date have demonstrated increased rates of Tp-e interval and Tp-e/QT in autoimmune diseases, including rheumatoid arthritis, systemic lupus erythematosus, and ankylosing spondylitis, exhibiting an intense systemic inflammation response. In addition, this increase was detected to be correlated with inflammatory parameters [35-37]. Some authors underlined the fact that this electrical abnormality could be a predictor of potential cardiac arrhythmias and cause predisposition. While the exact mechanism of the increase in the Tp-e interval and Tp-e/QT in patients with psoriasis vulgaris is not clearly established, we believe that the chronic inflammation process occurring in this disorder contributes to the ventricular repolarization heterogeneity. As a matter of fact, we found a significant correlation between the Tp-e time and the Tp-e/QT rate, and the hs-CRP level and the PASI score in patients with psoriasis vulgaris.

We did not assess the association between the ventricular arrhythmias and the Tp-e interval and Tp-e/QT ratio. Also, the study population could not be followed up prospectively for ventricular arrhythmic episodes. Thus, long-term follow-up and largescale prospective studies are needed to determine the arrhythmogenic risk and sudden cardiac death
(SCD) prediction value of prolonged Tp-e interval and increased Tp-e/QT ratio in psoriasis vulgaris patients. Finally, manual measurements of QT and Tp-e intervals on a paper-printed electrocardiogram is a potential limitation, and the patients being only Turkish in ethnicity, the collection of data from two centers and the absence of information related to the socioeconomic status of the patients might constitute an impediment in the adjustment of our results to the general population.

In conclusion, we detected a significant increase in the Tp-e interval and the Tp-e/QT rate in patients with psoriasis vulgaris. Additionally, the ventricular repolarization indices were observed to be associated with inflammation and the severity of the disease. In conclusion, the Tp-e interval and the Tp-e/QT rate could be considered as significant predictors of potential ventricular arrhythmia in patients with psoriasis vulgaris.

\section{Conflict of interest}

The authors declare no conflict of interest.

\section{References}

1. Gunes AT, Altıner D. History and epidemiology of psoriasis. Turkiye Klinikleri J Int Med Sci 2005; 1: 1-4.

2. Farber EM, Nall ML. The natural history of psoriasis in 5,600 patients. Dermatologica 1974; 148: 1-18.

3. Ryan C, Menter A. Psoriasis and cardiovascular disorders. G Ital Dermatol Venereol 2012; 147: 179-87.

4. Mehta NN, Azfar RS, Shin DB, et al. Patients with severe psoriasis are at increased risk of cardiovascular mortality: cohort study using the General Practice Research Database. Eur Heart J 2010; 31: 1000-6.

5. Simsek H, Sahin M, Akyol A, et al. Increased risk of atrial and ventricular arrhythmia in long-lasting psoriasis patients. Sci World J 2013; 2013: 901215.

6. Bicer A, Basar N, Karasu BB, et al. Increased QT dispersion in patients with psoriasis. Dicle Medical J 2010; 37: 223-7.

7. Antzelevitch C, Shimizu W, Yan GX, et al. Cellular basis for QT dispersion. J Electrocardiol 1998; 30: 168-75.

8. Kors JA, Ritsema van Eck HJ, van Herpen G. The meaning of the Tp-Te interval and its diagnostic value. J Electrocardiol 2008; 41: 575-80.

9. Antzelevitch C, Sicouri S, Di Diego JM, et al. Does TpeakTend provide an index of transmural dispersion of repolarization? Heart Rhythm 2007; 4: 1114-6.

10. Castro Hevia J, Antzelevitch C, Tornés Bárzaga F, et al. Tpeak-Tend and Tpeak-Tend dispersion as risk factors for ventricular tachycardia/ventricular fibrillation in patients with the Brugada syndrome. J Am Coll Cardiol 2006; 47: 1828-34.

11. Smetana P, Schmidt A, Zabel M, et al. Assessment of repolarization heterogeneity for prediction of mortality in cardiovascular disease: peak to the end of the T wave interval and nondipolar repolarization components. J Electrocardiol 2011; 44: 301-8.

12. Erikssen G, Liestøl K, Gullestad L, et al. The terminal part of the QT interval (T peak to $T$ end): a predictor of mortality after acute myocardial infarction. Ann Noninvasive Electrocardiol 2012; 17: 85-94. 
13. Watanabe N, Kobayashi Y, Tanno K, et al. Transmural dispersion of repolarization and ventricular tachyarrhythmias. J Electrocardiol 2004; 37: 191-200.

14. Gupta P, Patel C, Patel H, et al. T(p-e)/QT ratio as an index of arrhythmogenesis. J Electrocardiol 2008; 41: 567-74.

15. Louden BA, Pearce DJ, Lang W, et al. A Simplified Psoriasis Area Severity Index (SPASI) for rating psoriasis severity in clinic patients. Dermatol Online J 2004; 10: 7.

16. Day CP, McComb JM, Campbell RW. QT dispersion: an indication of arrhythmia risk in patients with long QT intervals. Br Heart J 1990; 63: 342-4.

17. Quinones MA, Otto CM, Stoddard M, et al. Recommendations for quantification of Doppler echocardiography: a report from the Doppler Quantification Task Force of the Nomenclature and Standards Committee of the American Society of Echocardiography. J Am Soc Echocardiogr 2002; 15: 167-84.

18. Devereux RB, de Simone G, Koren MJ, Roman MJ, Laragh $\mathrm{JH}$. Left ventricular mass as a predictor of development of hypertension. Am J Hypertens 1991; 4: 603S-7S.

19. Gelfand JM, Weinstein R, Porter SB, et al. Prevalence and treatment of psoriasis in the United Kingdom: a population-based study. Arch Dermatol 2005; 141: 1537-41.

20. Friedewald VE, Cather JC, Gelfand JM, et al. AJC editor's consensus: psoriasis and coronary artery disease. Am J Cardiol 2008; 102: 1631-43.

21. Mehta NN, Azfar RS, Shin DB, et al. Patients with severe psoriasis are at increased risk of cardiovascular mortality: cohort study using the General Practice Research Database. Eur Heart J 2010; 31: 1000-6.

22. Prodanovich S, Kirsner RS, Kravetz JD, et al. Association of psoriasis with coronary artery, cerebrovascular, and peripheral vascular diseases and mortality. Arch Dermatol 2009; 145: 700-3.

23. Klein RM, Vester EG, Brehm MU, et al. Inflammation of the myocardium as a trigger for arrhythmias. Z Kardiol 2000; 89 Suppl 3: 24-35.

24. Carvalho M, Soares R, Ribeiro F, et al. Rhythm profile in patients with psoriatic arthritis. Rev Port Cardiol 1990; 9: 311-7.

25. Markuszeski L, Bissinger A, Janusz I, et al. Heart rate and arrhythmia in patients with psoriasis vulgaris. Arch Med Res 2007; 38: 64-9.

26. Novikova DS, Korotaeva TV, Loginova E, et al. Clinical implication of assessment of heart rate variability in patients with psoriatic arthritis. Ter Arkh 2009; 81: 47-52.

27. Sarli B, Dogan Y, Baktir AO, et al. Heart rate recovery is impaired in patients with psoriasis. Med Princ Pract 2013; 22: 567-70.

28. Perkiomaki JS, Koistinen J, Yli-Mayry S, et al. Dispersion of QT interval in patients with and without susceptibility to ventricular tachyarrhytmias after previous myocardial infarction. J Am Coll Cardiol 1995; 26: 174-9.

29. Barr CS, Naas A, Freeman M, et al. QT dispersion and sudden unexpected death in chronic heart failure. Lancet 1994; 343: 327-9.

30. Opthof T, Coronel R, Janse MJ. Is there a significant transmural gradient in repolarization time in the intact heart? Repolarization gradients in the intact heart. Circ Arrythmia Electrophysiol 2009; 2: 89-96.

31. Lubinski A, Lewicka-Nowak E, Kempa M, et al. New insight into repolarization abnormalities in patients with congenital long QT syndrome. The increased transmural dispersion of repolarization. PACE 1998; 21: 172-5.

32. Shimizu M, Ino H, Okeie K, et al. T-peak to T-end may be a better predictor of high-risk patients with hypertrophic cardiomyopathy associated with a cardiac troponin I mutation than QT dispersion. Clin Cardiol 2002; 25: 335-9.

33. Zhao X, Xie Z, Chu Y, et al. Association between Tp-e/ QT ratio and prognosis in patients undergoing primary percutaneous coronary intervention for ST-segment elevation myocardial infarction. Clin Cardiol 2012; 35: 559-64.

34. Letsas KP, Weber R, Astheimer K, et al. Tpeak-Tend interval and Tpeak-Tend/QT ratio as markers of ventricular tachycardia inducibility in subjects with Brugada ECG phenotype. Europace 2010; 12: 271-4.

35. Avcı A, Demir K, Altunkeser BB, et al. Assessment of inhomogeneities of repolarization in patients with systemic lupus erythematosus. Ann Noninvasive Electrocardiol 2014; 19: 374-82.

36. Acar G, Yorgun H, Inci MF, et al. Evaluation of Tp-e interval and Tp-e/QT ratio in patients with ankylosing spondylitis. Mod Rheumatol 2014; 24: 327-30.

37. Acar G, Akkoyun M, Nacar BA, et al. Evatuation of Tp-e interval and Tp-e/QT ratio in patients with rheumatoid arthritis. Arch Turk Soc Cardiol 2014; 42: 29-34. 